\title{
Mechanical Calculation of Power Lines and Strands in Overhead Transmission Lines
}

\author{
Yongping $\mathrm{Li}^{1}, \mathrm{Yu} \mathrm{Liu}^{2}$ and Jibin $\mathrm{Zhang}^{3}$ \\ ${ }^{1}$ Computational Mechanics, Northeast Dianli University, 132012 Jilin, China \\ ${ }^{2}$ Civil Engineering, Northeast Dianli University, 132012 Jilin, China \\ 3 Transmission Engineering, Northeast Dianli University, 132012 Jilin, China
}

\begin{abstract}
This paper discusses the issue of shape and stress of overhead transmission lines. It fully considers that the conductor is subjected to axial force, shear, bending moment simultaneousiy. And the flat wire is basically in the elastic range. Because the stress is the strength of a certain point, the bending rigidity of the overhead lines can not be ignored. However, the catenary has been used in previous research and the force of the overhead lines is obtained on this basis. The author establishes a model of wire calculation under real condition, and gives a feasible simplified model. And then it gives the line shape formula, internal force formula, formula of stress in the strands. The results show that the original stress is significantly smaller.In this paper, the stress formula is almost close to the reality. It proves the reliability of the simplified model that the line shape includes the original flexible part.The results can not only fill the gaps in the theoretical study of the overhead lines, but also have some guidance to the production and design of overhead lines.
\end{abstract}

\section{Introduction}

So far the hypothesis has been adopted in the study of wire mechanics that line shape of overhead transmission line is catenary [1-5]. The conductor is only subjected to axial force, and the stress of the wire is replaced by the average stress. The average stress is equal to the axial force divided by the cross-sectional area of the wire. However, the real line shape of the hanging wire is not catenary. The real wire is rigid and it is subjected to axial force, shear force and bending moment unlike a cable that is just under tension. The previous hypothesis leads to the stress of the conductor far away from the actual stress, so that there are hidden dangers in the strength design. The purpose of this paper is to study the wire alignment under the natural suspended state, and the axial force, shear force and bending moment of the conductor under this condition. Next, the author obtains the line shape from the static equilibrium analysis, and thus finds out the internal force of the wire and the stress of the strands.

\section{Overhead line alignment}

Assume that the wire is regarded as a soft line in deformation due to excessive span. The assumption can simplify the analysis and calculation. And the catenary formula under this assumption [6] is easy to remember. Because the stress is the force received on the unit area, the bending rigidity of the overhead transmission lines is not small even in a very small wire. So bending stress should not be ignored.

\subsection{Alignment analysis}

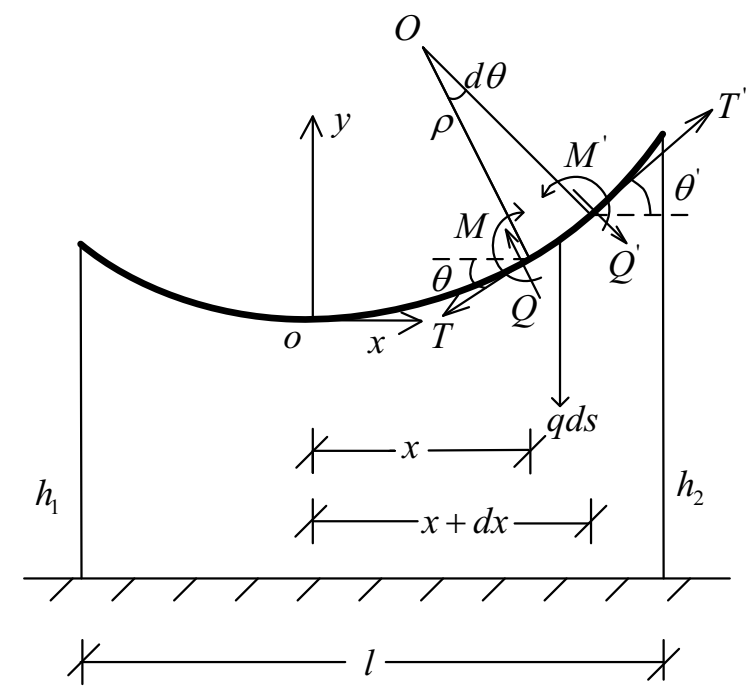

Figure 1. Natural hanging state diagram of wire in single span. Schematic diagram of force analysis of conductor. 
Figure 1 shows the natural state of single span wire in suspension. Select any wire element for mechanical analysis. The static equilibrium equations of the selected element are as follows.

$$
\begin{aligned}
& \left\{\begin{array}{c}
T^{\prime} \cos \theta^{\prime}-T \cos \theta+Q^{\prime} \sin \theta^{\prime}-Q \sin \theta=0 \\
T^{\prime} \sin \theta^{\prime}-T \sin \theta-Q^{\prime} \cos \theta^{\prime}+Q \cos \theta-q d s=0 \\
M^{\prime}-M+T \sin \theta d x-T \cos \theta d y-q d s \frac{d x}{2}=0
\end{array}\right. \\
& \left\{\begin{array}{c}
\frac{d M}{d s}=Q \\
\frac{d Q}{d s}=\frac{T}{\rho}-q \cos \theta \\
\frac{d T}{d s}=q \sin \theta-\frac{Q}{\rho}
\end{array}\right. \\
& \frac{d \rho}{d s} \frac{d^{2} M}{d s^{2}}+\rho \frac{d^{3} M}{d s^{3}}+\frac{d \rho}{d s} q \cos \theta+\frac{1}{\rho} \frac{d M}{d s}-q \sin \theta=0
\end{aligned}
$$

The differential relationship of moment, shear and axial force can be calculated by the formula (1). The formula (3) is obtained by the equation group (2). And the formula (3) is the static equilibrium line of the overhead lines considering the moment, shear and axial force. Because too complicated and difficult to solve, it is necessary to be simplified.

\subsection{Simplified alignment}

For most of the plain, the wire is relatively flat. It is considered within the elastic range. So the bending moment and curvature has the following relations in the formula (4) $[7,8]$.

$$
\begin{gathered}
\frac{1}{\rho}=\frac{M}{E I} \\
\frac{d^{3} \rho}{d \theta^{3}}-\frac{9}{\rho} \frac{d \rho}{d \theta} \frac{d^{2} \rho}{d \theta^{2}}+\frac{12}{\rho^{2}}\left(\frac{d \rho}{d \theta}\right)^{3}+\frac{d \rho}{d \theta}-a^{3} \rho^{3} \cos \theta \frac{d \rho}{d \theta}+2 a^{3} \rho^{4} \sin \theta=0
\end{gathered}
$$

Formula (5) was obtained by formula (4) into the formula (3). The formula (5) is a three order's, nonlinear, variable coefficient's and non-homogeneous equation. The equation's analytical solution is difficult to be obtained.

Because the wire is flat, its curvature radius changes very slowly along with the dip angle. Thus the ones are very small relative to the rest .They can be ignored that the two order derivative, third order derivative and the cubic of the first derivative of curvature radius and dip angle. And the corresponding coefficient is smaller than 1 . Ignore them and retain the main items. Simplified model is obtained by (6-1) or (6-2).

$$
\begin{gathered}
\frac{d \rho}{d \theta}-a^{3} \rho^{3} \cos \theta \frac{d \rho}{d \theta}+2 a^{3} \rho^{4} \sin \theta=0 \\
\frac{d \rho}{d \theta}=\frac{2 a^{3} \rho^{4} \sin \theta}{a^{3} \rho^{3} \cos \theta-1} \\
a^{3}=\frac{q}{E I}
\end{gathered}
$$

This is a first order's, nonlinear, variable coefficient's and non-homogeneous equation. Its analytical solution is (7).

$$
\cos \theta=\sqrt{\frac{\rho_{0}}{\rho}}\left(1+\frac{1}{5 a^{3} \rho^{3}}\right)-\frac{1}{5 a^{3} \rho^{3}}
$$

The formula (7) is the exact result of the simplified model. It is also the linear analytical solution. Although it is an implicit equation, it is one of the important results of this research. And it is so far unique result.

It is not difficult to prove that the first part of formula (7) is on behalf of the catenary, the second part is a correction term due to the influence of the shear and bending moment. Formula (7) contains the catenary, reflecting the rule that general contains special. It illustrates the rationality, scientificalness and credibility of the simplified method for the formula (3).

\subsection{Determining the phenotype of alignment}

Explicit formula of formula (7) is determined as follows.

$$
\begin{aligned}
& \rho=\rho_{0}+\Delta \rho, \\
& \frac{1}{\rho}=\frac{y^{\prime \prime}}{\left(1+y^{\prime 2}\right)^{3 / 2}},
\end{aligned}
$$

Because the $\frac{\Delta \rho}{\rho_{0}}$ is very small, the formula (8) is obtained.

$$
\left\{\begin{array}{l}
x=\frac{a^{3} \rho_{0}{ }^{4}}{1-a^{3} \rho_{0}{ }^{3}}\left[\theta+\sin \theta \cos \theta-\left(3-\frac{1}{a^{3} \rho_{0}{ }^{3}}\right) \sin \theta\right] \\
y=\frac{a^{3} \rho_{0}{ }^{4}}{1-a^{3} \rho_{0}{ }^{3}}\left[\theta+\sin ^{2} \theta-(1-\cos \theta)\left(3-\frac{1}{a^{3} \rho_{0}{ }^{3}}\right)\right]
\end{array}\right.
$$

Formula (8) is an explicit equation of overhead lines in general. Its coordinate system is shown in Figure 1. The lowest point of the wire is the origin. The right is the $\mathrm{X}$ axis and the up is the $\mathrm{Y}$ axis. The formula (8) is a parameter equation of $\rho_{0}$ and $\theta . \rho_{0}$ is the curvature radius of the lowest point of the overhead line. $\theta$ is the angle between the tangent direction and the horizontal direction of the wire.

\section{Mechanical calculation of overhead line}

\subsection{Internal force calculation of overhead line}

By formula (7), $\rho_{0}$ can be found in the determination of $\theta$. By the radius of curvature $\rho$, the internal force can be obtained under the assumption of elasticity.

1) moment,

$$
M=\frac{E I}{\rho}
$$


2) shear,

$$
Q=\frac{2 E I \rho q \sin \theta}{E I-q \rho^{3} \cos \theta}
$$

3) axial force,

$$
\begin{gathered}
T=\rho \frac{d Q}{d s}+\rho q \cos \theta=\frac{d Q}{d \theta}+\rho q \cos \theta \\
T=\rho q \cos \theta+2 \rho q \frac{\left(a^{3} \rho^{3}-1\right)\left(\cos \theta-a^{3} \rho^{3}\right)+2 a^{3} \rho^{3} \sin ^{2} \theta\left(1+2 a^{3} \rho^{3} \cos \theta\right)}{\left(a^{3} \rho^{3} \cos \theta-1\right)^{3}}
\end{gathered}
$$

At the lowest point of the wire $(\theta=0)$, its axial force is seen in the formula (11-3).

$$
T_{0}=\rho_{0} q+\frac{2 \rho_{0} q}{a^{3} \rho^{3}-1}=\rho_{0} q\left(1-\frac{2}{a^{3} \rho^{3}-1}\right)
$$

The former represents the axial force of the span wire. The latter is an additional term due to the influence of bending moment and shear. It is proved that the true axial force of the wire is lower than that of the flexible wire.

Table 1.Internal force table

\begin{tabular}{|c|c|}
\hline & auther \\
\hline Axial force $(\mathrm{N})$ & 21463 \\
\hline Shear force $(\mathrm{N})$ & 0.000165 \\
\hline $\begin{array}{c}\text { Bending moment } \\
(\mathrm{N} \bullet \mathrm{mm})\end{array}$ & 771.82 \\
\hline
\end{tabular}

Numerical value in table 1 is obtained by the wire of LGJ400/35 in elevation suspension state. Assumed that the span is equal to $545 \mathrm{~m}$ and the sag is equal to $23 \mathrm{~m}$. The internal force is obtained at the point where $\theta=10^{\circ}$. The cross section of LGJ400/35 is shown in Figure 2. Parameters of LGJ400/35 are shown in Table 2.

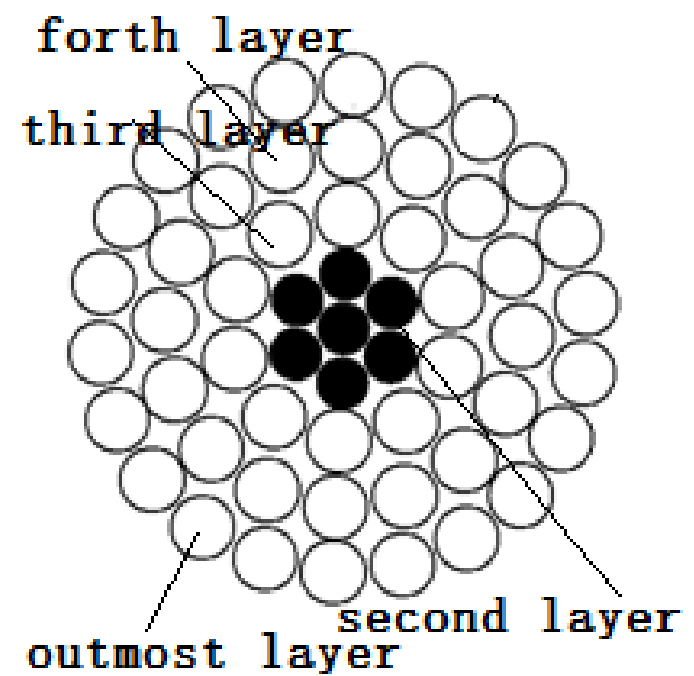

Figure 2. Cross section of LGJ400/35.
Table 2. Parameters of LGJ400/35.

\begin{tabular}{|c|c|c|c|}
\hline$d_{s}(m m)$ & $d_{a}(m m)$ & $E_{s}\left(\mathrm{~N} / \mathrm{mm}^{2}\right)$ & $E_{a}\left(\mathrm{~N} / \mathrm{mm}^{2}\right)$ \\
\hline 2.50 & 3.22 & 202000 & 68000 \\
\hline
\end{tabular}

$d_{s}$ is diameter of steel core and $d_{a}$ is diameter of aluminum strand.

\subsection{Stress of strand calculation of overhead line}

The premise of wire breaking is the strands breaks.And the first break of the strands is the outermost strand. So this paper analyzes the axial normal stress of the most outer strand. The axial normal stress is normal referred to as the strand stress. It is obvious that there are two kinds of stresses. One is caused by the axial tension of the wire. The other is caused by the bending of the wire. The total stress of the strands is $\sigma=\sigma_{l}+\sigma_{M}$, not the empirical formula $\sigma_{1}=\frac{T}{A}$ [1].

3.2.1 The stress of the strand caused by the axial tension of the wire

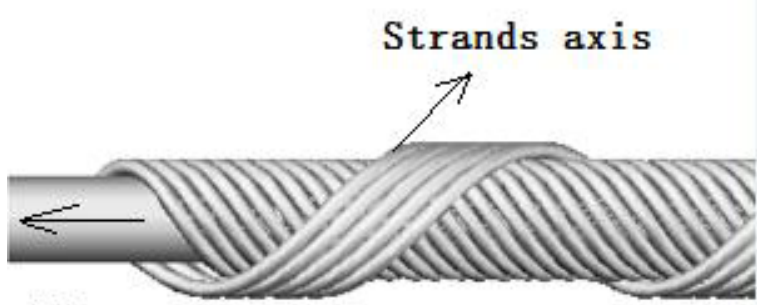

Wire axis

Figure 3. Diagram of the wire axis and the srrands axis.

The strain of the strand is along the direction of the strand, not the axial direction of the wire. The outermost strand stress is showed in formula (12).

$$
\begin{gathered}
\sigma_{l}=E_{a} \varepsilon_{l}=E_{a}\left(\cos ^{2} \alpha_{n}-\mu_{n} \sin ^{2} \alpha_{n}\right) \varepsilon_{u} \\
\varepsilon_{u}=\frac{T}{E A}
\end{gathered}
$$

$E_{a}$ is the elastic modulus of the aluminum strand. ${ } \varepsilon_{l}$ is the axial strain of the strands and $\varepsilon_{u}$ is the axial strain of the wire. $\alpha_{n}$ is the winding angle of strands. $\mu_{n}$ is the Poisson's ratio of the wire and it can be regarded as equal to ${ }^{\mu}$ [9]. T is the axial tension of the wire and EA is the tensile stiffness of the conductor section. 


$$
\sigma_{l}=\frac{T E_{a}}{E A}\left(\cos ^{2} \alpha_{n}-\mu_{n} \sin ^{2} \alpha_{n}\right)
$$

\subsubsection{The stress of the strand caused by wire bending}

The bending of a wire is an objective existence. There is a formula (4) in the elastic range. So section normal stress is as follows.

$$
\sigma_{M}^{\prime}=\frac{E y}{\rho}=\frac{E_{a} M\left(D-d_{n}\right)}{2 E I}
$$

$E_{a}$ is the elastic modulus of the aluminum strand. dn is the diameter of the aluminum strand. $\mathrm{M}$ is the bending moment of the wire. D is diameter of conductor section and $\mathrm{EI}$ is bending rigidity of conductor section.

Considering the angle between the wire axis and the strand, the stress in the strand is showed in formula (16).

$$
\sigma_{M}=\frac{E_{a} M\left(D-d_{n}\right)}{2 E I}\left(\cos ^{2} \alpha_{n}-\mu_{n} \sin ^{2} \alpha_{n}\right)
$$

\subsubsection{The total stress of the strand by the superposition of the two stresses}

$$
\sigma=\sigma_{l}+\sigma_{M}=E_{a}\left[\frac{T}{E A}+\frac{M\left(D-d_{n}\right)}{2 E I}\right]\left(\cos ^{2} \alpha_{n}-\mu_{n} \sin ^{2} \alpha_{n}\right)
$$

It is obvious that the stress caused by stretching and bending is greater than one received tension only. The growth rate is e. EI and EA are respectively representing the tensile stiffness and the flexural rigidity of the cross section [10].

Table 3.Stress of strands.

\begin{tabular}{|c|c|}
\hline Outermost layer (N/mm) & 134.26 \\
\hline Forth layer (N/mm) & 45.30 \\
\hline Third layer (N/mm) & 45.35 \\
\hline Second layer (N/mm) & 45.39 \\
\hline
\end{tabular}

The basic assumption of the table 3 is the same as 3.1 .

$$
e=\frac{M\left(D-d_{n}\right)}{2 E I} \cdot \frac{E A}{T} \times 100 \%
$$

Because $M T \approx E I q$ at the lowest point in the wire, the ratio is showed as follows.

$$
e=\frac{q\left(D-d_{n}\right)}{2 T^{2}} \cdot E A \times 100 \%
$$

The error rate is not negligible because it is generally more than $20 \%$. It can reach $35 \%$ or even higher in the large cross-section wire. However, the flexible conductor just ignores the bending stress.

Moreover there is no formula of stress in the catenary.
Occasionally it is replaced by the stress of the rod. That is not scientific. Because the elastic modulus of steel strands is almost 3 times higher than the elastic modulus of aluminum strands. Stress is the force of a certain point, and it must be respectively calculated either the stresses of the steel strands or the aluminum strands. Otherwise the error is very large.

\section{Conclusion}

In this paper, explicit and implicit linear formulas of the overhead lines are obtained by using the method of differential calculus and elastic mechanics. The linear formula is derived by taking into account that the conductor is subjected to bending moment, shear force and axial force simultaneously. And on this basis, the paper carries out the analysis of the internal force of the wire, the analysis of the stress of the strands and the calculation of the conductor sag. Finally they are truly expressed, that the linear, stress and sag in the natural state of suspension. This paper not only makes up the theoretical gaps in the field of the mechanical calculation of wire, but also provides the modeling basis and the analysis method for the research of the related problems. It has a certain guiding significance for transmission line construction, but also provides a theoretical basis for the design and production of wires.

\section{References}

1. S.-M.. Meng, Wei. Kong, Overhead transmission line design (China Electric Power Press, China Beijing, 2007)

2. D.-S. Zhang, Design Handbook for high voltage power supply circuit of electric power engineering (China Electric Power Press, China Beijing, 2004)

3. B.-Z. Li, High voltage overhead power transmission line construction calculation principle (China Electric Power Press, China Beijing, 2008)

4. G.-Z. Jiang, China. Science Technology and Engineering 8, 8 (2008)

5. X. Wei, S.-Z.. Qiang, China. Building Science Research of Sichuan 29, 4 (2003)

6. W.-H. Zhang, J.-B. Yu, China. Electric Power Construction 33, 2 (2012)

7. X.-F. Sun, X.-Y. Fang, L.-T. Guan, mechanics of materials (Higher Education Press, China Beijing, 2006)

8. S. P. Timoshenko, mechanics of materials (Sciencec Press, China Beijing, 1978)

9. Y.-P. Li, X.-F. Yun, W.-J. Li, China. Electric Power Construction 10, 10 (1999)

10. Y.-P. Li, Z.-Y. Huang, Y. Ni, China. Water Resources and Power 30, 2 (2012) 